\title{
Simultaneous gastric adenosquamous carcinoma and gastric carcinoma with lymphoid stroma: A case report
}

\author{
HIROHIKO MIYAKE ${ }^{1}$, CHIKA MIYASAKA ${ }^{1}$, MITSUAKI ISHIDA ${ }^{1}$, \\ HIROKAZU MIKI $^{2}$, KENTAROU INOUE ${ }^{2}$ and KOJI TSUTA ${ }^{1}$ \\ Departments of ${ }^{1}$ Pathology and Laboratory Medicine, and ${ }^{2}$ Surgery, Kansai Medical University, Hirakata, \\ Osaka 573-1010, Japan
}

Received December 11, 2018; Accepted April 30, 2019

DOI: $10.3892 / \mathrm{mco} .2019 .1860$

\begin{abstract}
Primary gastric adenosquamous carcinoma (ASC) is a rare tumor, and gastric carcinoma with lymphoid stroma (GCLS) is a distinct and relatively rare tumor, which is characterized histopathologically by prominent lymphoid infiltration and is commonly associated with Epstein-Barr virus (EBV) infection. The association between ASC and GCLS has been poorly understood, and only two cases of concomitant occurrence of ASC and GCLS have been reported previously. In this report, we describe the first documented case of simultaneous gastric ASC and GCLS. A 58-year-old Japanese male complained of loss of appetite. Gastric endoscopic examination revealed a type 3 tumor in the antrum. Distal gastrectomy with lymph node dissection was performed after neoadjuvant chemotherapy. Macroscopic examination of the resected specimen revealed two discontinuous lesions in the antrum. The first lesion, an ASC, was detected preoperatively by endoscopic examination. This type 3 tumor was composed of a mixture of squamous cell carcinoma ( $\sim 75 \%$ of the tumor) and poorly-to-moderately differentiated adenocarcinoma ( 25\%). No EBV-encoded RNA (EBER)-positive neoplastic cells were noted. The tumor had invaded into the serosa. The second lesion was a GCLS, which was not preoperatively detected by endoscopic examination. The neoplastic cells of the GCLS were EBER-positive. The tumor had invaded into the muscularis propria. Accordingly, a diagnosis of simultaneous gastric ASC (pT4a) and GCLS (pT2) was made. The pathogenesis of gastric ASC has been under debate. Only limited cases of ASC associated with EBV have been reported. Notably, EBV has not been associated with the development of an ASC component in the previously reported concomitant
\end{abstract}

Correspondence to: Dr Mitsuaki Ishida, Department of Pathology and Laboratory Medicine, Kansai Medical University, 2-5-1 Shinmachi, Hirakata, Osaka 573-1010, Japan

E-mail: ishidamt@hirakata.kmu.ac.jp

Key words: gastric cancer, adenosquamous carcinoma, carcinoma with lymphoid stroma, Epstein-Barr virus, double cancer cases of ASC and GCLS, as well as in the present case study. Therefore, additional studies are required to clarify the pathogenesis of gastric ASC, including its association with EBV.

\section{Introduction}

Primary gastric adenosquamous carcinoma (ASC) is a rare, aggressive tumor, and accounts for less than $1 \%$ of gastric carcinomas (1). Gastric ASC is characterized histopathologically by the presence of adenocarcinoma and squamous cell carcinoma (SCC) components within the same tumor (1). Recently, the clinicopathological characteristics of gastric ASC have been analyzed (1). This type of tumor mainly affects males (73.3\%). In a previous study, half of tumors were stage T4 and most patients $(86.2 \%)$ had lymph node metastasis (1). These results suggest that gastric ASC has an aggressive clinical course compared with conventional gastric adenocarcinoma. However, because of rarity of the tumor, pathogenesis of this type of tumor remains unclear.

Gastric carcinoma with lymphoid stroma (GCLS) is a distinct and relatively rare histopathological variant that is characterized by prominent lymphoid infiltration within and around the neoplastic cells, and is commonly associated with Epstein-Barr virus (EBV) infection (2). It has been reported that this type of tumor shows a relatively better prognosis compared with conventional gastric adenocarcinoma (2).

The association between ASC and GCLS has been poorly understood, and only two reports of concomitant occurrence of ASC and GCLS have been reported $(3,4)$. In this article, we described a case of simultaneous gastric ASC and GCLS, and assessed the clinicopathological features of the previously reported cases, as well as the present one, and the pathogenesis of gastric ASC, including the association with EBV infection in gastric ASC.

\section{Case report}

A 58-year-old Japanese male without significant past history complained of loss of appetite. Gastric endoscopic examination revealed a type 3 tumor in the antrum, and biopsy of the lesion showed adenocarcinoma with squamous cell carcinoma 

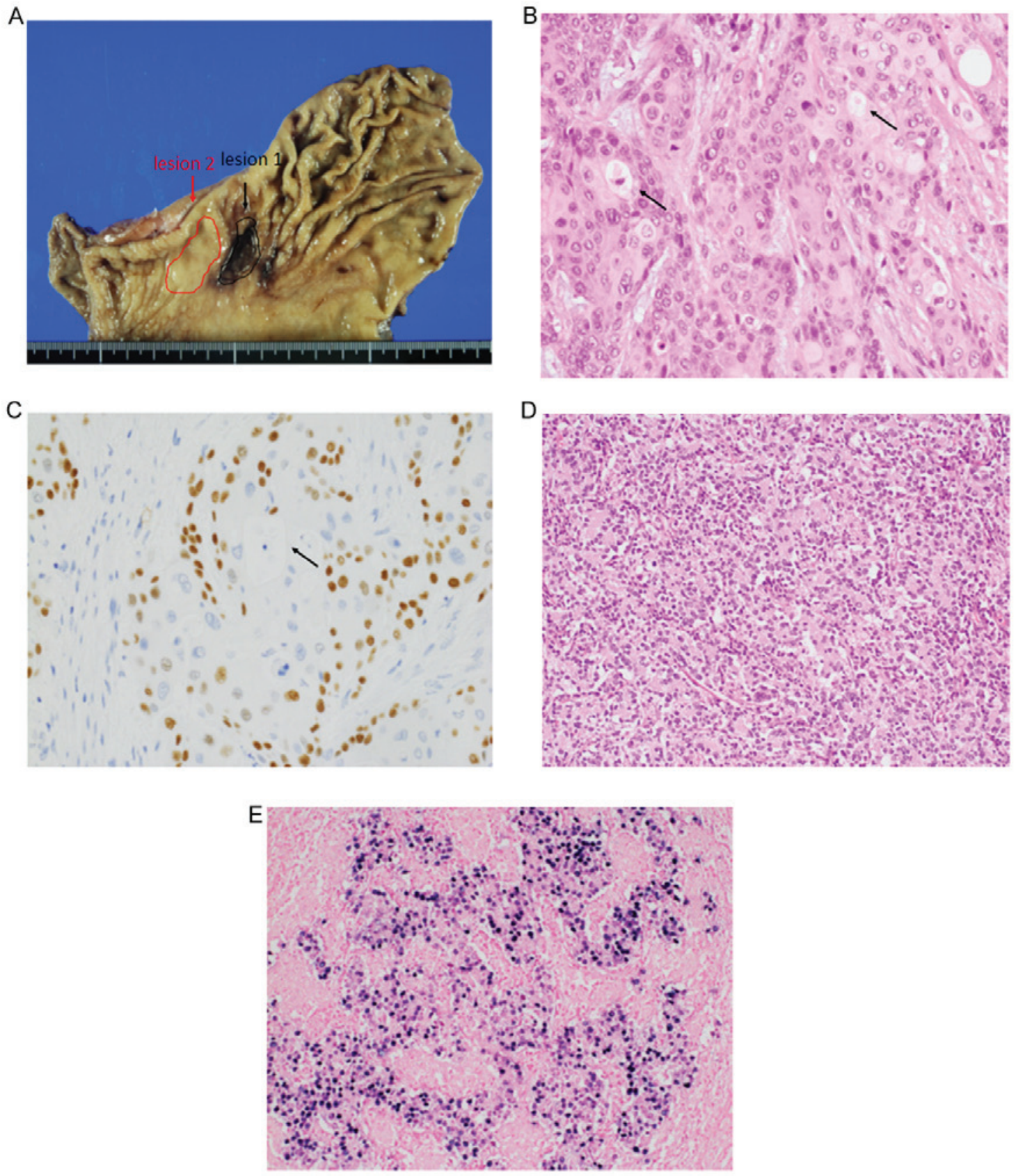

Figure 1. Macroscopic, histopathological, immunohistochemical and in situ hybridization findings of the gastric tumors. (A) Macroscopic findings. Type 3 tumor (black, lesion 1) and submucosal tumor-like type 1 tumor (red, lesion 2) are closely located, but no continuity is present. (B) Histopathological features of adenosquamous carcinoma (lesion 1). Squamous cell carcinoma component is composed of solid nest or trabecular proliferation of the neoplastic cells with relatively rich eosinophilic cytoplasm and large round to oval nuclei. Focal glandular formation is noted (arrows) (H\&E; magnification, x400). (C) Immunostain for p40 demonstrating positive reactivity in the squamous cell carcinoma component. The neoplastic cells showing glandular formation lacking p40 expression (arrow; magnification, x400). (D) Gastric carcinoma with lymphoid stroma (lesion 2). Diffuse proliferation of the neoplastic cells accompanying abundant lymphocytic infiltration (H\&E; magnification, x200). (E) EBER is detected in the neoplastic cells of the gastric carcinoma with lymphoid stroma (magnification, $\mathrm{x} 200)$. EBER, Epstein-Barr virus encoding RNA.

(SCC) component. Laparoscopic examination revealed disseminated lesions in the peritoneum; thus, he underwent neoadjuvant chemotherapy (11 cycles of S-1 and oxaliplatin), followed by distal gastrectomy with lymph node dissection. The post-operative course was uneventful, and chemotherapy was added. The patient has been free from tumor recurrence during 8 months of medical follow-up.

Formalin-fixed and paraffin-embedded specimens of the resected tumor were processed for routine histological examination and immunohistochemical and in situ hybridization analyses. Immunohistochemical analyses were performed using autostainers (XT System Benchmark, Roche Diagnostics, Basel, Switzerland and link 48, DAKO Cytomation, Glostrup, Denmark). The primary antibodies used were a rabbit monoclonal antibody against caudal type homeobox 2 (cdx-2) (EPR2764Y, Roche), a mouse monoclonal antibody against keratin 5/6 (D5/16 B4, DAKO), a mouse monoclonal antibody against keratin 7 (OV-TL 12/30, DAKO), a mouse monoclonal antibody against keratin 20 (Ks20.8, DAKO), and a mouse monoclonal antibody against 
Table I. Clinicopathological features of gastric adenosquamous carcinoma with carcinoma with lymphoid stroma.

\begin{tabular}{|c|c|c|c|c|c|c|c|c|c|}
\hline Factor & Age/sex & $\begin{array}{c}\text { No. of } \\
\text { the lesions }\end{array}$ & Location & Size (mm) & p63/p40 & EBER & Prognosis & $\begin{array}{l}\text { Authors, } \\
\text { year }\end{array}$ & (Refs.) \\
\hline Case 1 & 50 's/male & 1 & Upper & $90 \times 85$ & $\begin{array}{l}\text { Not } \\
\text { done }\end{array}$ & $\begin{array}{c}\operatorname{SCC}(-), \\
\operatorname{GCLS}(+)\end{array}$ & $\begin{array}{l}\text { AWD, } \\
10 \text { years }\end{array}$ & $\begin{array}{c}\text { Oya et al, } \\
2010\end{array}$ & (3) \\
\hline Case 2 & $67 /$ male & 1 & Upper & $60 \times 50$ & $\begin{array}{c}\operatorname{Ad}(-), \\
\operatorname{SCC}(+), \\
\operatorname{GCLS}(-)\end{array}$ & $\begin{array}{l}\operatorname{Ad}(-), \operatorname{SCC}(-) \\
\operatorname{GCLS}(+)\end{array}$ & $\begin{array}{c}\text { Not } \\
\text { available }\end{array}$ & $\begin{array}{l}\text { Kuroda et al, } \\
\quad 2010\end{array}$ & (4) \\
\hline Present case & $58 /$ male & 2 & Lower & $\begin{array}{l}35 \times 25 \\
(\mathrm{ASC}) \\
45 \times 36 \\
(\mathrm{GCLS})\end{array}$ & $\begin{array}{c}\operatorname{Ad}(-), \\
\operatorname{SCC}(+), \\
\operatorname{GCLS}(-)\end{array}$ & $\begin{array}{c}\operatorname{Ad}(-), \\
\operatorname{SCC}(-) \\
\operatorname{GCLS}(+)\end{array}$ & $\begin{array}{c}\text { AWD, } \\
8 \text { months }\end{array}$ & - & - \\
\hline
\end{tabular}

Ad, adenocarcinoma; ASC, adenosquamous carcinoma; AWD, alive without disease; EBER, Epstein-Barr virus encoding RNA; GCLS, gastric carcinoma with lymphoid stroma; SCC, squamous cell carcinoma.

p40 (BC28, Roche). In situ hybridization analysis was performed using autostainer (XT System Benchmark, Roche Diagnostics, Basel).

Macroscopic examination of the resected specimen revealed two discontinuous lesions in the antrum (Fig. 1A). The first lesion, an ASC, was detected preoperatively by endoscopic examination. This type 3 tumor was composed of a mixture of SCC (approximately 75\% of the tumor) and poorly to moderately differentiated adenocarcinoma (approximately 25\%) (Fig. 1B). The neoplastic cells of the SCC component had large round to oval nuclei with small nucleoli and relatively rich eosinophilic cytoplasm (Fig. 1B). Although obvious keratinization was not detected, an intercellular bridge was noted. This component was intermingled with adenocarcinoma component, which formed irregular fused glands and had large round to oval nuclei (Fig. 1B). The tumor invaded into the serosa, and lymphovascular invasion was noted. Immunohistochemically, the SCC component was diffusely positive for $\mathrm{p} 40$, but the adenocarcinoma component was negative for p40 (Fig. 1C). Keratin 5/6 was expressed only in the SCC component, and keratin 7 and 20, and cdx-2 were not expressed in both components. In situ hybridization detected no EBV-encoded RNA (EBER)-positive neoplastic cells in both components. The lymph nodes contained metastatic ASC (with intermingled SCC and adenocarcinoma components) (5/39).

The second lesion, a GCLS, was not preoperatively detected by endoscopic examination (Fig. 1A). This submucosal tumor-like type 1 tumor was composed of diffuse proliferation of the neoplastic cells containing large round to oval nuclei with nucleoli accompanying dense lymphocytic infiltration with lymphoid follicle formation (Fig. 1D). The tumor had invaded into the muscularis propria, and lymphovascular invasion was noted. Immunohistochemical analyses revealed that the neoplastic cells were focally positive for cdx-2 and keratin 20, but negative for p40, and keratin 5/6 and 7. EBER was detected in the neoplastic cells by in situ hybridization (Fig. 1E). The lymph nodes contained metastatic GCLS (5/39).

Accordingly, a diagnosis of double gastric cancers composed of ASC (pT4a) and GCLS (pT2) was made. Moreover, both tumors showed mild chemotherapeutic effect.

\section{Discussion}

We herein report the first documented case of double gastric cancers composed of ASC and GCLS. The peculiar finding of the present case was the presence of two discontinuous tumors composed of ASC and GCLS.

Table I summarizes the clinicopathological features of the two previously reported cases of concomitant ASC and GCLS, as well as the present one. In the case reported by Oya et al, the adenocarcinoma component of the ASC was a GCLS (3). In the other case reported by Kuroda et al, both GCLS and ASC were present within the same tumor (the adenocarcinoma component of the ASC was a conventional adenocarcinoma); thus, the authors suspected two possibilities regarding the pathogenesis of the lesion: i) collision of two distinct tumors, and ii) differentiation of adenocarcinoma toward an EBV-related tumor and SCC (4). Although ASC and GCLS were close in location in the present case, the pathogenetic relationship between the two lesions is unclear.

The pathogenesis of gastric ASC has been under debate, and several hypotheses have been proposed: i) squamous metaplastic transformation of adenocarcinoma, ii) oncogenic transformation of metaplastic squamous cells, iii) collision of concurrent adenocarcinoma and SCC, and iv) differentiation of stem cells toward glandular and squamous cells (2). In the present case, the adenocarcinoma and SCC components were intermingled, as they were in the metastatic lymph nodes. Furthermore, it has been reported that adenocarcinoma is the most frequent component of the metastatic lymph nodes of patients with gastric ASC, followed by a combination of both adenocarcinoma and SCC components (2). Therefore, the first hypothesis is the most considerable.

It has been well recognized that the occurrence of GCLS is associated with EBV in most cases. Although only limited cases of ASC associated with EBV have been reported (5), the SCC components of the two previously reported cases and the present case were not associated with EBV infection. Therefore, additional studies are needed to clarify the clinicopathological features and pathogenesis of gastric ASC, including the association with GCLS. 


\section{Acknowledgements}

Not applicable.

\section{Funding}

No funding was received.

\section{Availability of data and materials}

All data generated or analyzed during the present study are included in this published article.

\section{Authors' contributions}

$\mathrm{CM}$ and $\mathrm{MI}$ were involved in the conception and design of the study. HMiy, CM, MI, HMik, KI and KT were involved in the acquisition and analysis of data. CM and MI drafted the manuscript and constructed the figure. The final version of the manuscript has been read and approved by all authors.

\section{Ethics approval and consent to participate}

This study was conducted in accordance with the Declaration of Helsinki.

\section{Patient consent for publication}

Patient consent was obtained according to our hospital policy.

\section{Competing interests}

The authors declare that they have no competing interests.

\section{References}

1. Feng F, Zheng G, Qi J, Xu G, Wang F, Wang Q, Guo M, Lian X and Zhang $\mathrm{H}$ : Clinicopathological features and prognosis of gastric adenosquamous carcinoma. Sci Rep 7: 4597, 2017.

2. Lim H, Park YS, Lee JH, Son DH, Ahn JY, Choi KS, Kim DH, Choi KD, Song HJ, Lee GH, et al: Features of gastric carcinoma with lymphoid stroma associated with Epstein-Barr virus. Clin Gastroenterol Hepatol 13: 1738-1744, 2015.

3. Oya H, Taniguchi H, Koyama T, Sekine S, Tsuta K, Kushima R and Katai $\mathrm{H}$ : Adenosquamous carcinoma, with a component of carcinoma with lymphoid stroma positive for EBER-1, a case report. Stomach Intestine 45: 1987-1991, 2010 (In Japanese).

4. Kuroda N, Oonishi K, Inoue K, Ohara M, Mizuno K and Lee GH: Lymphoepithelioma-like carcinoma of the stomach associated with adenosquamous carcinoma. Med Mol Morphol 43: 170-173, 2010.

5. Ribeiro J, Oliveira A, Malta M, Oliveira C, Silva F, Galaghar A, Afonso LP, Neves MC, Medeiros R, Pimentel-Nunes P and Sousa $\mathrm{H}$ : Clinical and pathological characterization of Epstein-Barr virus-associated gastric carcinomas in Portugal. World J Gastroenterol 23: 7292-7302, 2017. 